\title{
FIRST-based survey of compact steep spectrum sources
}

\section{Multifrequency VLBA observations of very compact objects}

\author{
M. Kunert-Bajraszewska ${ }^{1}$, A. Marecki ${ }^{1}$, and P. Thomasson ${ }^{2}$ \\ 1 Toruń Centre for Astronomy, N. Copernicus University, 87-100 Torun, Poland \\ e-mail: amr@astro.uni.torun.pl \\ 2 Jodrell Bank Observatory, University of Manchester, Macclesfield, Cheshire SK11 9DL, UK
}

Received 27 October 2005 / Accepted 7 January 2006

ABSTRACT

Context. Evidence has been mounting recently that activity in some radio-loud AGNs (RLAGNs) can cease shortly after ignition and that perhaps even a majority of very compact sources may be short-lived phenomena because of a lack of stable fuelling from the black hole. Thus, they can fade out before having evolved to large, extended objects. Re-ignition of the activity in such objects is not ruled out.

Aims. With the aim of finding more examples of these objects and to investigate if they could be RLAGNs switched off at very early stages of their evolution, multifrequency VLBA observations of six sources with angular sizes significantly less than an arcsecond, yet having steep spectra, have been made.

Methods. Observations were initially made at $1.65 \mathrm{GHz}$ using the VLBA with the inclusion of Effelsberg telescope. The sources were then re-observed with the VLBA at 5, 8.4 and $15.4 \mathrm{GHz}$. All the observations were carried out in a snapshot mode with phase referencing.

Results. One of the sources studied, 0809+404, is dominated by a compact component but also has diffuse, arcsecond-scale emission visible in VLA images. The VLBI observations of the "core" structure have revealed that this is also diffuse and fading away at higher frequencies. Thus, the inner component of 0809+404 could be a compact fading object. The remaining five sources presented here show either core-jet or edge-brightened double-lobed structures indicating that they are in an active phase.

Conclusions. The above result is an indication that the activity of the host galaxy of 0809+404 may be intermittent. Previous observations obtained from the literature and those presented here indicate that activity had ceased once in the past, then restarted, and has recently switched off again.

Key words. radio continuum: galaxies - galaxies: active - Galaxy: evolution

\section{Introduction}

The activity period for radio-loud AGNs (RLAGN) can last up to $\sim 10^{8}$ years (Alexander \& Leahy 1987; Liu et al. 1992) and, as their lobes are huge reservoirs of energy, even if the energy supply from the central engine to the hotspots and the lobes eventually cuts off, the radio sources are still observable for a substantial period of time. This so-called "coasting phase" of the lobes of a RLAGN can last up to $10^{8} \mathrm{yr}$ (Komissarov \& Gubanov 1994; Slee et al. 2001) and preserves information of past nuclear activity. As the source gradually fades out, its spectrum becomes steeper and steeper because of radiation and expansion losses. Objects possessing these features are sometimes termed "relics" or "faders".

The structure and other properties of the double radio source B2 0924+30, identified with an E/S0 galaxy IC 2476, shows it to be a good example of a fader (Cordey 1987).
The projected linear size of the whole system is $270 h^{-1} \mathrm{kpc}^{1}$ which indicates that it is a Large Symmetric Object (LSO). Jamrozy et al. (2004) have confirmed that B2 0924+30 is indeed a relic radio structure and that it switched off its activity $\sim 5 \times 10^{7}$ years ago and, as such, can be labelled a "dead" radio galaxy. A large number of examples of fossil radio galaxies or cluster relic systems have recently been found by Cohen et al. (2004) as a result of their VLA observations at $74 \mathrm{MHz}$.

There are no obvious reasons for the existence of a lower limit to the length of the activity period in a RLAGN; it could be shorter than has already been seen for LSOs. If this is the case it could be that the growth of the radio source has been impeded, even at an early stage of its evolution. As a result,

\footnotetext{
${ }^{1}$ For consistency with earlier papers in this field, the following cosmological parameters have been adopted throughout this paper: $H_{0}=$ $100 \mathrm{~km} \mathrm{~s}^{-1} \mathrm{Mpc}^{-1}$ and $q_{0}=0.5$. Wherever in the text the linear sizes are referred to, $h^{-1}$ is introduced.
} 
small-scale faders might exist. An attempt to test observationally if there are young faders among Medium-sized Symmetric Objects (MSOs), i.e. the objects that have linear sizes in the range $1-20 h^{-1} \mathrm{kpc}$, has been made by Kunert-Bajraszewska et al. (2005) - hereafter Paper II. This revealed one strong candidate $-1542+323$.

Giroletti et al. (2005) have described a class of low power compact (LPC) radio sources, their small sizes and moderate luminosities (comparable to those of low power giant FR I radio galaxies) being ascribed to a number of different physical reasons: youth, low kinetic power of the jets or frustration and also the premature end of nuclear activity. $1855+37$, one of the sources they investigated, could be a very good example of a compact fader.

There is no reason why one should not search for faders among the most compact of radio sources, particularly those belonging to the class of Compact Symmetric Objects (CSOs), i.e. sub-kiloparsec-scale extragalactic radio sources with symmetric radio structures. Most of these sources are triples with the central component being a radio core, or doubles with only two detectable radio lobes. Some CSOs have radio spectra that peak at a few gigahertz and have been classified as Gigahertz-Peaked Spectrum (GPS) sources. GPS radio galaxies seem to be CSOs with a simple structure (O'Dea 1998). However, most GPS quasars with a core-jet or complex milliarcsecond-scale morphology do not seem to have symmetric structures and they are thought to be a different class of object (Stanghellini 2003).

The physical origin of CSOs has been explained in two ways. According to the frustration scenario (van Breugel et al. 1984) the radio source is permanently confined to a region within the host galaxy by a dense environment from which it is impossible for it to evolve into a large source. Alexander (2000) also claims that the observational density of sources in the power-linear size plane can be reproduced only if it is assumed that there exists a class of young frustrated objects. They would remain very weak and have diffuse hotspots and a brighter jet. Those objects, which manage to escape the highdensity regions, would continue their evolution and reach the classical FR II stage.

Alternatively, Phillips \& Mutel (1982) and Carvalho (1985) suggested that CSOs - they were labelled compact-doubles at that time; the term "CSO" was introduced later by Wilkinson et al. (1994) and Readhead et al. (1994) - could be young radio sources that would evolve into large radio objects during their lifetimes. Based upon this scenario, Readhead et al. (1996) proposed an evolutionary scheme unifying three classes of radio sources: CSOs, MSOs - a subset of the Compact Steep Spectrum sources (CSS) class - and LSOs.

At the present time this is the youth scenario that is generally accepted; see the review by Fanti (2000). An argument in favour of this has been found mainly in age measurements of individual classes of radio sources: CSOs are younger than $\sim 10^{4}$ years (Owsianik et al. 1998; Giroletti et al. 2003), MSOs are typically $\sim 10^{5}$ years old (Murgia et al. 1999) and LSOs can manifest their activity for up to $\sim 10^{8}$ years (Alexander \& Leahy 1987; Liu et al. 1992). Snellen et al. (1999, 2000) added an important ingredient to this scheme, namely that the radio luminosities of CSOs increase as they evolve, reach a maximum in the MSO phase and then gradually decrease as these objects increase in size to become LSOs.

Marecki et al. (2003b, 2006a) claim that this evolutionary track is not the only one possible. In fact, several, if not a "continuum" of such tracks might exist and the one shown by Snellen et al. $(1999,2000)$ just appears as the only one simply because of selection effects. If the energy supply cuts off early, the object leaves the "main sequence" proposed by Snellen et al. (1999, 2000) and will never reach the LSO stage, at least in a given phase of activity. Thus, there should exist a class of (very) small-scale objects that resemble large-scale faders. This conforms to early predictions that many CSOs could be young objects that switch off after a short period of time (Readhead et al. 1994). Strong support for such an idea also comes from Reynolds \& Begelman (1997) who proposed a model in which extragalactic radio sources are intermittent on timescales of $\sim 10^{4}-10^{5}$ years. The above considerations have recently been supported by observational results obtained by Gugliucci et al. (2005), suggesting that many CSOs die young or are episodic in nature, and so it is likely that only a minority of them "survive" and further evolve.

In this paper - the fourth of the series - VLBA observations of 6 compact sources which are candidates for prematurely dying CSOs are presented and discussed.

\section{The observations and data reduction}

A sample of 60 candidates which could be weak Compact Steep Spectrum sources was selected from the VLA FIRST catalogue (White et al. 1997) ${ }^{2}$. The selection criteria have been given by Kunert et al. (2002) - hereafter Paper I. All the sources were initially observed with MERLIN at $5 \mathrm{GHz}$ and the results of these observations led to the selection of several groups of objects for further study with MERLIN and the VLA (Paper II), as well as the VLBA and the EVN (Marecki et al. 2006a, hereafter Paper III). One of those groups contained six sources that were barely resolved by MERLIN at $5 \mathrm{GHz}$, but still had steep $\left(\alpha \leq-0.5, S \propto v^{\alpha}\right)$ spectra between 1.4 and $4.85 \mathrm{GHz}$. The basic properties of the six sources are given in Table 1 .

Initial 1.65-GHz VLBA observations of the sources listed in Table 1 were carried out on 27 and 28 July 2002 in a snapshot mode with phase referencing. The Effelsberg telescope was also included in order to improve the resolution at that comparatively low frequency. Each target source scan was interleaved with a scan on a phase reference source throughout an $\sim 8$-h track. The total cycle time (target and phase reference) was 9 min including telescope drive times, with $\sim 7$ min actually on the target source per cycle.

After a careful inspection of the $1.65-\mathrm{GHz}$ images, it was decided that all the sources except one should be observed at 5, 8.4 and $15.4 \mathrm{GHz}$. The exception was $1502+291$, for which it was considered that only an observation at $5 \mathrm{GHz}$ would be necessary to confirm its core-jet structure, indicating that it was not a candidate for a "switched off" object. These follow-up VLBA-only observations were carried out on 13 and

\footnotetext{
${ }^{2}$ Official website: http://sundog.stsci.edu
} 
Table 1. Optical magnitudes and radio flux densities of target sources at two frequencies.

\begin{tabular}{|c|c|c|c|c|c|c|c|c|c|c|c|}
\hline $\begin{array}{c}\text { Source } \\
\text { name } \\
(1)\end{array}$ & $\begin{array}{c}\text { RA } \\
\text { h m s } \\
\text { (2) }\end{array}$ & $\begin{array}{l}\text { Dec } \\
\circ, \prime \prime\end{array}$ & (4) & $m_{R}$ & (6) & $\begin{array}{c}\text { Total } \\
\text { flux at } \\
1.4 \mathrm{GHz} \\
{[\mathrm{mJy}]} \\
(7)\end{array}$ & $\begin{array}{c}\log P_{1.4 \mathrm{GHz}} \\
{\left[\begin{array}{c}\mathrm{W} \mathrm{Hz} \\
(8)\end{array}\right]}\end{array}$ & $\begin{array}{c}\text { Total } \\
\text { flux at } \\
4.85 \mathrm{GHz} \\
{[\mathrm{mJy}]} \\
(9)\end{array}$ & $\alpha_{1.4 \mathrm{GHz}}^{4.85 \mathrm{GHz}}$ & $\begin{array}{c}\text { [mas] } \\
(11)\end{array}$ & $\begin{array}{c}\text { LLS } \\
\qquad \begin{array}{c}\left.h^{-1} \mathrm{pc}\right] \\
(12)\end{array}\end{array}$ \\
\hline $0809+404$ & 081253.131 & 401900.09 & $\mathrm{Q}$ & 19.50 & 0.551 & 1068 & 26.63 & 392 & -0.81 & 14.87 & 54.9 \\
\hline $0949+287$ & 095206.090 & 282832.35 & G & 20.15 & - & 1364 & $27.38 *$ & 529 & -0.76 & 308.79 & $1330.0 *$ \\
\hline $1159+395$ & 120149.982 & 391911.26 & G & 23.32 & 2.370 & 599 & 27.78 & 249 & -0.71 & 41.08 & 161.3 \\
\hline $1315+396$ & 131718.653 & 392528.02 & Q & 18.20 & 1.560 & 615 & 27.39 & 227 & -0.80 & 33.77 & 143.8 \\
\hline $1502+291$ & 150426.715 & 285430.55 & $q$ & 18.58 & $0.056 ?$ & 567 & $24.29 ?$ & 261 & -0.63 & 41.60 & $30.8 ?$ \\
\hline $1616+366$ & 161823.546 & 363201.33 & G & 19.35 & 0.734 & 536 & 26.60 & 268 & -0.56 & $\sim 60.00$ & 242.0 \\
\hline
\end{tabular}

Column (1): source name in the IAU format; Col. (2): source right ascension (J2000) extracted from FIRST; Col. (3): source declination (J2000) extracted from FIRST; Col. (4): optical identification: G - galaxy, Q - quasar, q - star-like object without known redshift; Col. (5): red magnitude extracted from SDSS/DR4; Col. (6): redshift; Col. (7): total flux density at $1.4 \mathrm{GHz}$ extracted from FIRST; Col. (8): Log of radio luminosity at $1.4 \mathrm{GHz}$ in $\mathrm{W} \mathrm{Hz}^{-1}$; Col. (9): total flux density at $4.85 \mathrm{GHz}$ extracted from GB6; Col. (10): spectral index between 1.4 and 4.85 GHz calculated using flux densities in Cols. (7) and (9); Col. (11): largest Angular Size (LAS) in milliarcseconds measured in the 1.65-GHz VLBA image - in most cases, as a separation between the outermost component peaks; in one case (denoted with “ ") measured in the image contour plot; Col. (12): largest Linear Size (LLS) in $h^{-1}$ pc. Values denoted with “*” were computed assuming median redshift $z=1.085$. Redshift quoted for $1502+291$ is the redshift of Abell 2022 cluster.

14 October 2003. (Only 9 telescopes were used for the observations due to the failure of VLBA-KP antenna.) Each target source scan was interleaved with a scan on a phase reference source throughout an $\sim 12$-h track. The total cycle time (target and phase reference) was 8 min including telescope drive times, with $\sim 6$ min actually on the target source per cycle at each frequency. Except for $1502+291$, the cycles for a given targetcalibrator pair were grouped and rotated round the three frequencies. Only two sources $(1315+396,1616+366)$ have been detected at $15.4 \mathrm{GHz}$.

The $u-v$ coverage for the observations of the source $0809+404$ at all four frequencies, that are typical for all the observations, are shown in Fig. 1.

The whole data reduction process was carried out using AIPS. The data obtained during the second observational campaign (at 5, 8.4 and $15.4 \mathrm{GHz}$ ) were affected by Earth Orientation Parameter (EOP) errors introduced by the VLBA correlator. These errors were first removed in the reduction process. Residual fringe delay and rate corrections derived for the appropriate phase-reference sources, were applied to the corresponding target source data and initial images of these were produced. For a majority of the target sources, these images and their corrected data were then used as input parameters for further cycles of phase self-calibration. In most cases amplitude self-calibration was also applied. The final "naturally weighted" images were produced using IMAGR. The total intensity images are shown in Figs. 2 to 7. Flux densities of the principal components of the sources were measured using the AIPS task JMFIT and are listed in Table 2.

It was realised that, because of poor $u-v$ coverage and a "break-up" of the structure of some sources at the higher frequencies, appreciable amounts of flux density could be missing. Therefore, spectral index maps that were tentatively produced were not considered to be reliable and it was decided not to include them, nor the integrated spectra in this paper.
Also the spectral indices one can calculate from the flux densities quoted in Table 2 should only be treated as a coarse approximation.

In addition to the observations described above, an unpublished $15-\mathrm{GHz}$ VLA observation of $0809+404$, made in A-conf. by B. Clark and R. Perley in November 1983 as a part of investigations of the B3-VLA sample (Vigotti et al. 1989), has been included here with the authors' kind permission (Fig. 2, upper left panel).

\section{Comments on individual sources}

0809+404. VLA observations at 4.9 and $8.5 \mathrm{GHz}$ by Fanti et al. (2001) - hereafter F2001 - show the source to have a highly asymmetric double structure with the two components separated by 1 .' 2 and with a flux density ratio of $\sim 100: 1$ at $4.9 \mathrm{GHz}$. The much weaker western component is somewhat resolved with the VLA at $8.5 \mathrm{GHz}$ and, not surprisingly, is not visible in the VLBA image of Dallacasa et al. (2002) - hereafter D2002 at $1.67 \mathrm{GHz}$, nor in our VLBA image at approximately the same frequency (Fig. 2), that looks very similar to the image of D2002. The eastern component, compact at VLA resolutions at the lower frequencies, has been resolved with the VLBA at $1.67 \mathrm{GHz}$ into a structure that could be a double. However, this may just be an artefact of the contouring and D2002 consider it to have an amorphous structure. Our VLBA images at $5 \mathrm{GHz}$ and $8.4 \mathrm{GHz}$ (Fig. 2) and our inability to detect the source in our 15.4-GHz VLBA observations, confirm the latter. The source is simply fading away at the higher frequencies and there is no indication of a core or hotspots. The tongue of emission pointing to the south-west in the $1.65-\mathrm{GHz}$ image is also seen in the image of D2002, although the rather extended emission at its extremity in our image, and which is absent in that of D2002, is probably an artefact resulting from more extended emission that is present and being resolved. 

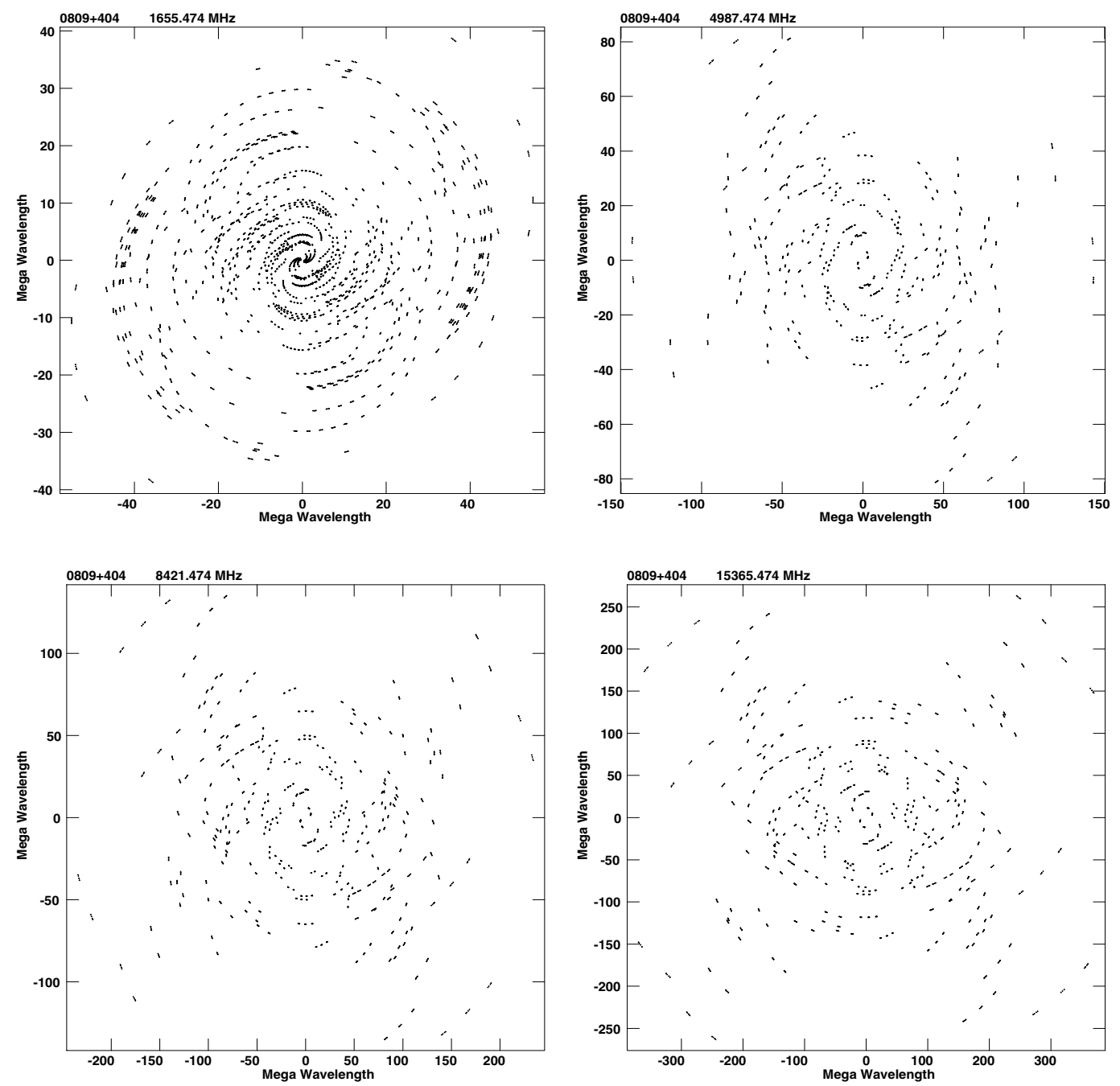

Fig. 1. Typical VLBA $u-v$ coverage at four different frequencies attained during the observations of $0809+404$.

The 15-GHz VLA (Fig. 2) observations show only the brighter eastern component which remains mostly unresolved and there is no hint of a radio core in this image. The total flux density of this component at $15 \mathrm{GHz}$ is $146.8 \mathrm{mJy}$, which yields a spectral index $\alpha=-1.03$ between 8.5 and $15 \mathrm{GHz}$.

Based upon the value of the spectral index between 1.4 and $4.85 \mathrm{GHz}(\alpha=-0.81)$ and the total flux densities at those frequencies (see Table 1), the interpolated total flux density of the source at $1.65 \mathrm{GHz}$ is $932 \mathrm{mJy}$. This means that $48 \%$ of the total flux density has been missed in our VLBA image which can be partially explained by the fact that only the brighter component in the VLA images made by F2001 has been detected. Moreover, compared with the flux densities of the brighter component of the source at 4.9 and $8.5 \mathrm{GHz}$ measured by F2001, it appears that only $\sim 47 \%$ and $\sim 28 \%$ of its flux density has been detected at the two corresponding frequencies in our VLBA observations.

According to Vigotti et al. (1997) 0809+404 is a Seyfert galaxy with redshift $z=0.551$. A galaxy is also the (automated) morphological identification of this object $\left(\mathrm{RA}=8^{\mathrm{h}} 12^{\mathrm{m}} 53^{\mathrm{s}} .109\right.$, Dec $=+40^{\circ} 19^{\prime} 0$. $^{\prime} 00$ ) included in Data Release 4 of the Sloan
Digital Sky Survey (SDSS/DR4), the latest release of SDSS at the time of writing. The redshift quoted by SDSS is in a full agreement with that of Vigotti et al. (1997). However, spectroscopically 0809+404 appears in SDSS as a quasar which is perhaps a more appropriate identification given that 0809+404 is included in the list of type-II quasar candidates (Zakamska et al. 2003) - see further discussion in Sect. 4.2.

0949+287. Our VLBA radio images show a double structure for this source (Fig. 3), the two detected components appearing to be radio lobes with steep spectra between 1.65 and $8.4 \mathrm{GHz}$. There is a compact feature in the eastern radio lobe, that is becoming weaker at the higher frequencies. The more diffuse western lobe also contains a small compact component with a spectrum which is becoming slightly flatter towards higher frequencies and so is most probably a hotspot. The two features on the northern edge of the western lobe in the $1.65 \mathrm{GHz}$ image are probably artefacts. There is no indication of a core in any of the images.

The assumed total flux density at $1.65 \mathrm{GHz}$ is $1201 \mathrm{mJy}$ which indicates that $\sim 4 \%$ of the total flux density of the source has not been seen because of resolution. The optical object 

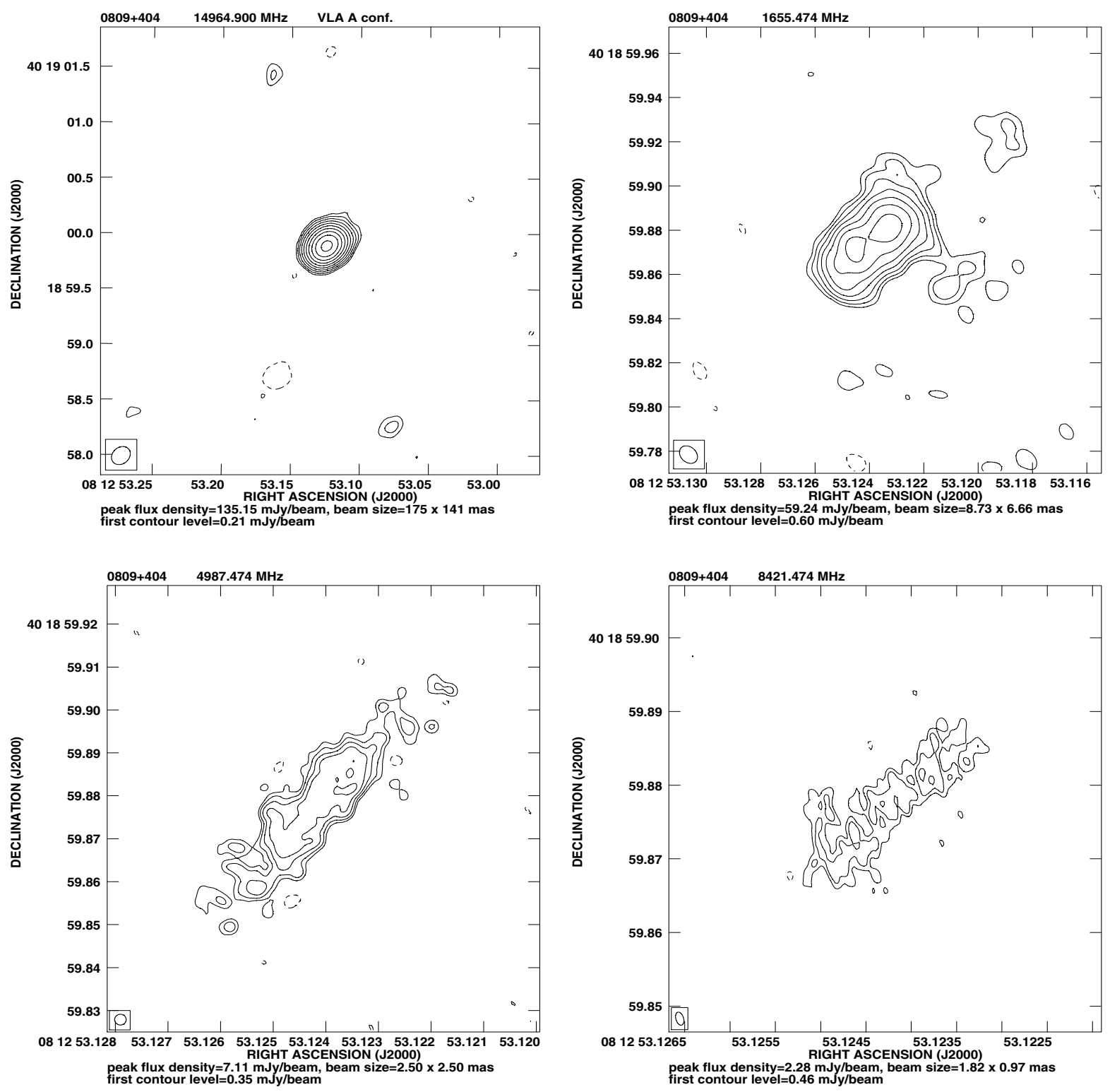

Fig. 2. The VLA (A-conf.) $15 \mathrm{GHz}$ map (upper left) and VLBA maps of 0809+404 at 1.65, 5 and $8.4 \mathrm{GHz}$. Contours increase by a factor 2 and the first contour level corresponds to $\approx 3 \sigma$.

extracted from SDSS/DR4 is a galaxy and its position (RA = $9^{\mathrm{h}} 52^{\mathrm{m}} 66^{\mathrm{s}} 098$, Dec $=+28^{\circ} 28^{\prime} 32^{\prime \prime} .40$ ) is marked with a cross on all maps. It is to be noted that there is another galaxy with $m_{R}=$ 21.74 located 2"'5 south of the galaxy identified with the radio source.

The double structure of $0949+287$ has been recently confirmed by $22 \mathrm{GHz}$ VLA observations made by Bolton et al. (2004) and its integrated spectrum shown there confirms it to be a compact steep spectrum source. The polarisation properties of $0949+287$ are not known.

$1159+395$. This source has been identified as a galaxy with a redshift $z=2.37$ (F2001). A galaxy is also the (automated) morphological identification of this object included in SDSS/DR4 $\left(\mathrm{RA}=12^{\mathrm{h}} 01^{\mathrm{m}} 50^{\mathrm{s}} .002\right.$, Dec $\left.=+39^{\circ} 19^{\prime} 10^{\prime} \cdot 95\right)$, although the redshift is not given. Our $1.65,5$ and $8.4-\mathrm{GHz}$ images show that the source has a double structure, the two components of which are clearly radio lobes oriented in a north-south direction (Fig. 4). The spectral indices of the lobes between 1.65 and $5 \mathrm{GHz}$ and between 5 and $8.4 \mathrm{GHz}$ are very steep. The elongated structure aligned east-west in the northern lobe in the $1.65-\mathrm{GHz}$ image is probably an artefact. The only indication of a core in any of the images is in the $5-\mathrm{GHz}$ image, in which there is a peak of emission at $\mathrm{RA}=$ $12^{\mathrm{h}} 01^{\mathrm{m}} 49^{\mathrm{s}} \cdot 965$, Dec $=+39^{\circ} 19^{\prime} 11^{\prime \prime} 028$. However, this is very doubtful as there is no indication of a core at this position in the other images. The double structure of the source has been confirmed in 1.65-GHz (D2002) and 5-GHz (Orienti et al. 2004) VLBA images and it has been classified as a CSO. The interpolated total flux density at $1.65 \mathrm{GHz}$ is $533 \mathrm{mJy}$, so our VLBA image accounts for $\sim 81 \%$ of the total flux density. 

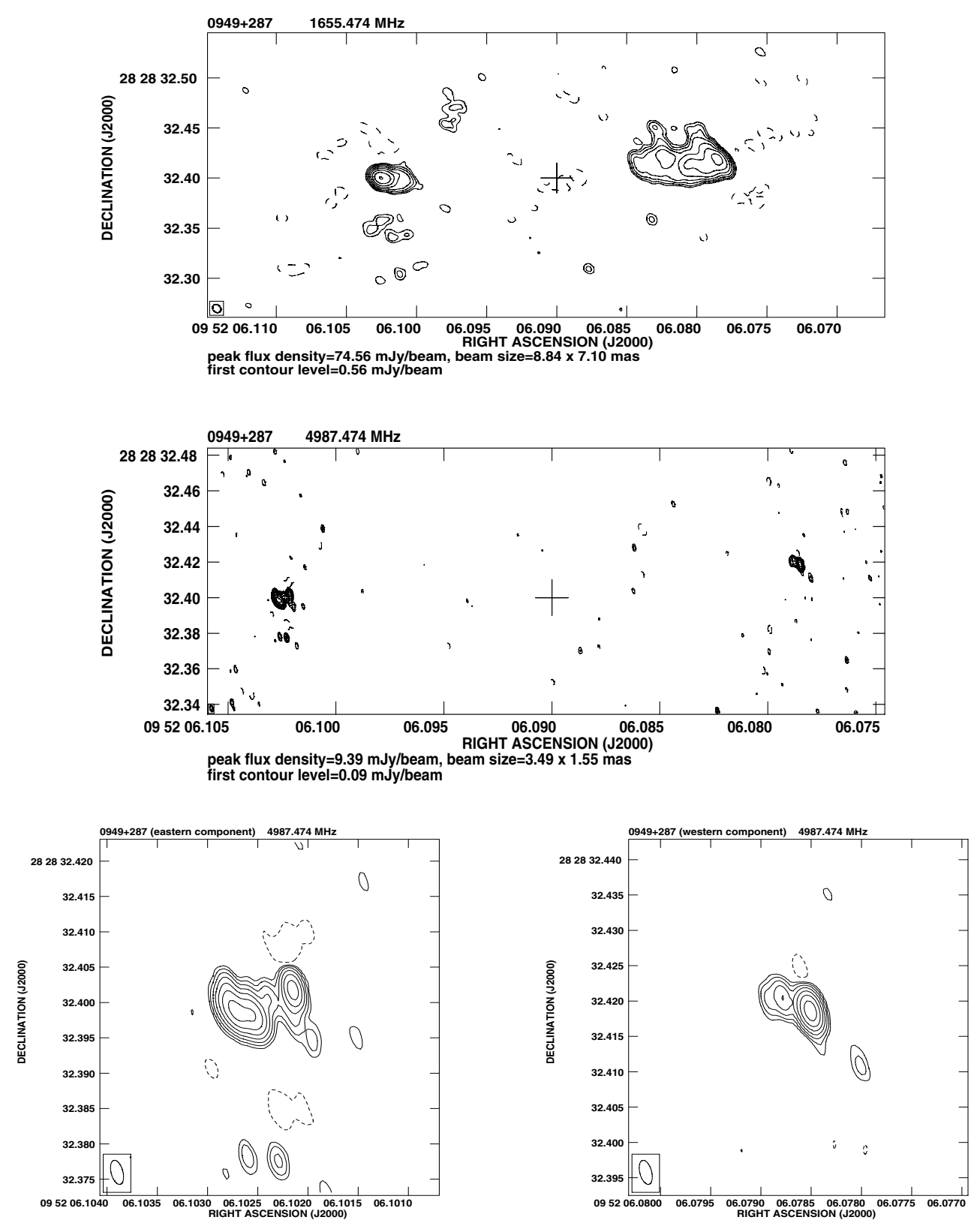

Fig. 3. The VLBA maps of $0949+287$ at $1.65 \mathrm{GHz}$ (top) and $5 \mathrm{GHz}$ (middle). The bottom row of panels show enlargements of the main components of the source at $5 \mathrm{GHz}$. Contours increase by a factor 2 and the first contour level corresponds to $\approx 3 \sigma$. The position of the optical object extracted from SDSS/DR4 is marked with a cross.

1315+396. The VLBA images indicate this to be a core-jet source (Fig. 5) that has been detected at all four frequencies. The spectrum of the core component is steepening towards higher frequencies. The $1.65-\mathrm{GHz}$ image accounts for $\sim 46 \%$ of the total flux density of the source $(538 \mathrm{mJy})$, the latter being derived from the flux densities and spectral index between 1.4 and $4.85 \mathrm{GHz}$. The diffuse jet structure, visible in the $1.65-\mathrm{GHz}$ image, could be partially resolved by the VLBA. The source has been optically identified with a quasar with a redshift $z=1.56$ (Vigotti et al. 1990). According to SDSS/DR4, $1315+396$ is a star-like object with unknown redshift at the position: $\mathrm{RA}=13^{\mathrm{h}} 17^{\mathrm{m}} 18^{\mathrm{s}} .643$, Dec $=+39^{\circ} 25^{\prime} 28^{\prime \prime} .16$.
1502+291. The VLBA images of this source primarily show an asymmetric structure directed to the north-east (Fig. 6). As this structure was already clearly visible in the $1.65-\mathrm{GHz}$ image prior to the higher frequency observations, the source had already been rejected as a candidate for a dying CSO and consequently was only observed at $5 \mathrm{GHz}$ to determine its spectral index and to confirm its core-jet nature. The peak of emission at $\mathrm{RA}=15^{\mathrm{h}} 04^{\mathrm{m}} 26^{\mathrm{s}} .696$, Dec $=+28^{\circ} 54^{\prime} 30^{\prime} .545$ (Table 2) has a flat spectrum and is a radio core. Approximately $70 \%$ of the total flux density $(510 \mathrm{mJy})$ of $1502+291$ has been seen in our 1.65-GHz image. VLBA snapshot observations of $1502+291$ at 2.3 and $8.4 \mathrm{GHz}$ were also carried out as a part of the 

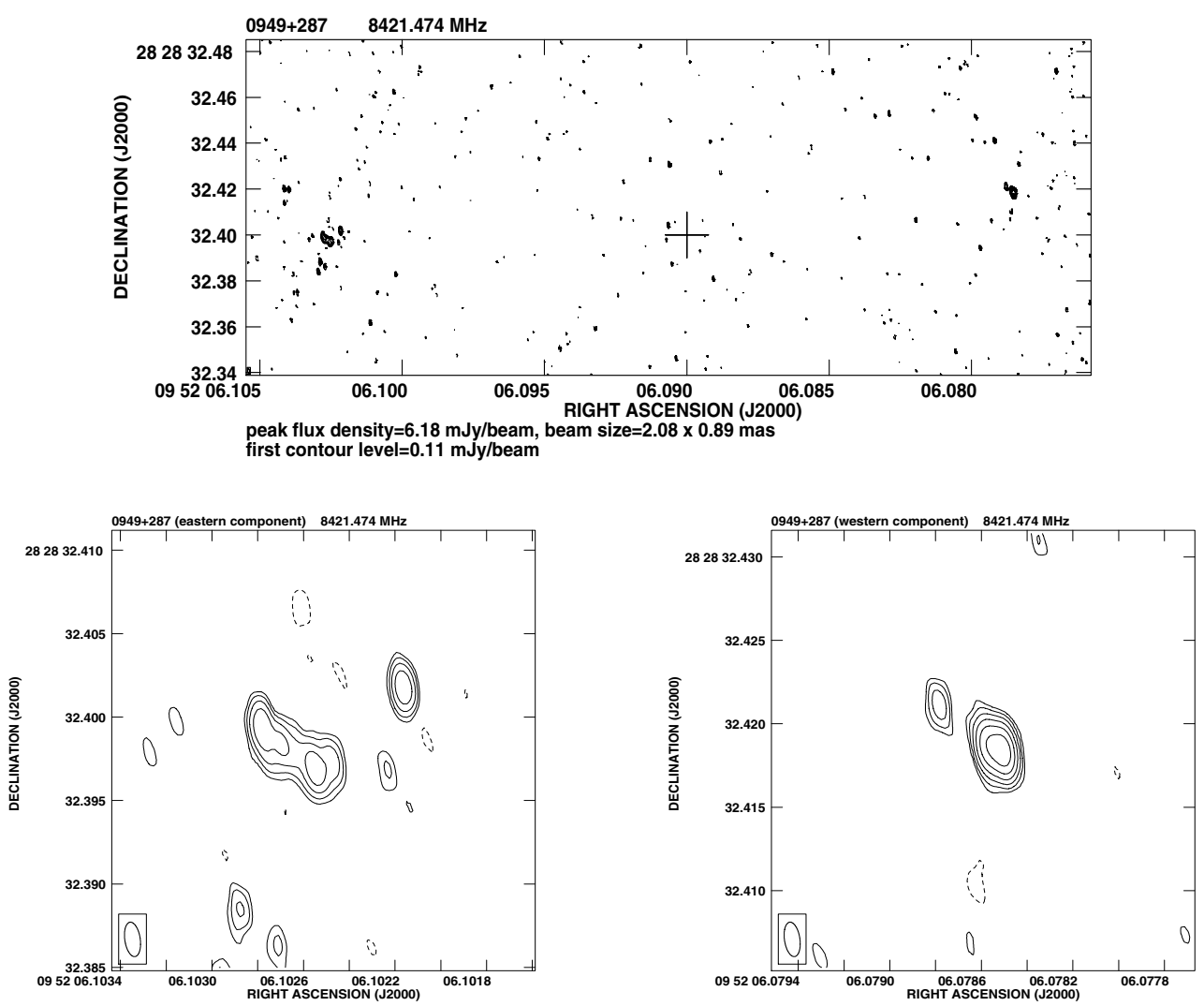

Fig. 3. continued. The VLBA map of 0949+287 (top) and its enlarged components (bottom) at $8.4 \mathrm{GHz}$. Contours increase by a factor 2 and the first contour level corresponds to $\approx 3 \sigma$. The position of the optical object extracted from SDSS/DR4 is marked with a cross.

VLBA Calibrator Survey (VCS1, Beasley et al. 2002). The $2.3 \mathrm{GHz}$ image shows a core-jet structure whereas at $8.4 \mathrm{GHz}$ only the core is visible.

Of interest in the $1.65-\mathrm{GHz}$ image of $1502+291$ are two regions of diffuse emission located to the south-east of the corejet object. It could be that they are lobes of a very nearby (with respect to the "main" core-jet structure), relic, compact double system. The flux density of the better defined, more southerly lobe has been measured (Table 2). These putative lobes of a dead source, are invisible in the 5-GHz image, so they must have steep spectra. Full-track VLBI observations are necessary to confirm the conjecture that there are two double sources in the field of $1502+291.1502+291$ is a member of cluster of galaxies (Abell 2022) and, based on this, an estimate of its redshift $(z=0.056)$ has been made (Abell et al. 1989). According to SDSS/DR4, 1502+291 is a star-like object with unknown redshift at the position: $\mathrm{RA}=15^{\mathrm{h}} 04^{\mathrm{m}} 26.698$, Dec $=$ $+28^{\circ} 54^{\prime} 30^{\prime} 54$.

1616+366. A published VLA image of this radio galaxy at $8.4 \mathrm{GHz}$ as a part of the Jodrell Bank-VLA Astrometric Survey (JVAS, Patnaik et al. 1992) shows it to have a core-jet structure directed to the south-west. The 1.65-GHz VLBA image (Fig. 7) shows an elongated structure that appears as a corejet object at higher frequencies. The radio core at the position $\mathrm{RA}=16^{\mathrm{h}} 18^{\mathrm{m}} 23^{\mathrm{s}} .581$, Dec $=+36^{\circ} 32^{\prime} 01^{\prime \prime} .813$ has a flat spectrum between 1.65 and $15.4 \mathrm{GHz}$. The interpolated total flux density at $1.65 \mathrm{GHz}$ is $488 \mathrm{mJy}$, so our VLBA image accounts for only $\sim 14 \%$ of the total flux density. This percentage is higher at $8.4 \mathrm{GHz}$ where our VLBA image accounts for $\sim 57 \%$ of the total flux density compared with the VLA image made by Patnaik et al. (1992).

$1616+366$ is present in SDSS/DR4 and has been identified as a galaxy with a redshift of $z=0.734$ located at: RA = $16^{\mathrm{h}} 18^{\mathrm{m}} 23^{\mathrm{s}} .582$, Dec $=+36^{\circ} 32^{\prime} 01^{\prime \prime} .75$.

\section{Discussion}

\subsection{CSOs with arcsecond-scale relic extensions}

According to Polatidis \& Conway (2003) and references therein, CSOs are young radio sources as their kinematic ages are of the order of $10^{3}-10^{4}$ years. Recently, Gugliucci et al. (2005) have investigated the ages of CSOs in a systematic manner and have shown that there is a clear cutoff in the age distribution at approximately 500 years, suggesting that CSOs may be young, not only because they are in the initial stages in an evolutionary chain, but that they are also short-lived i.e. their activity phase lasts for only a few hundred years. The most straightforward cause of this is a lack of stable fuelling. It follows that, because of a cutoff of the energy transport from the core to the lobes, not only does the luminosity of the source drop, but also diffuse radio lobes showing an absence of edge-brightening result as the hotspots fade away quickly. 

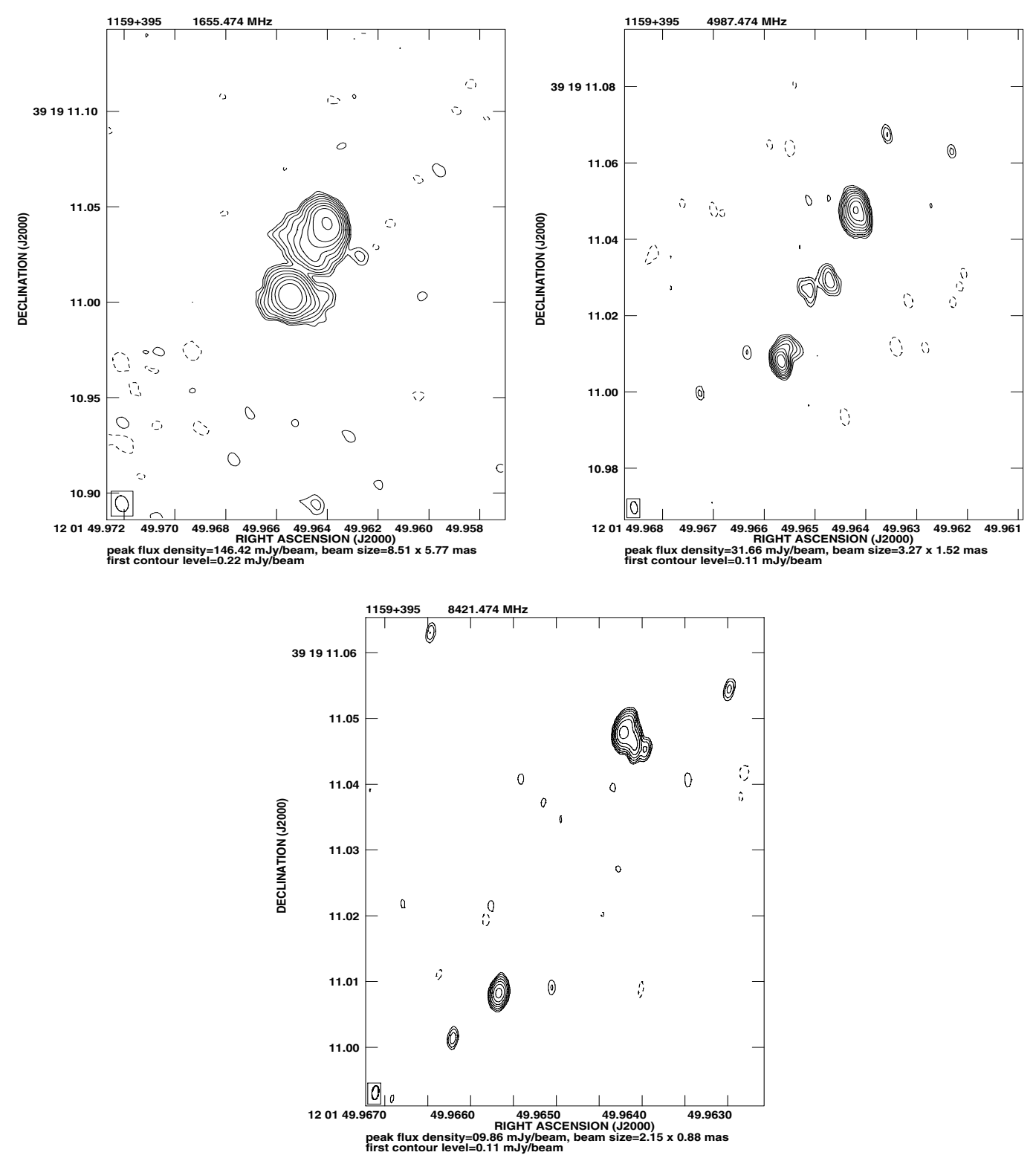

Fig. 4. The VLBA maps of $1159+395$ at $1.65,5$ and $8.4 \mathrm{GHz}$. Contours increase by a factor 2 and the first contour level corresponds to $\approx 3 \sigma$.

It could be that low frequency observations might reveal remnants of earlier stages of activity as in the case of $0108+388$, which is a compact double with an arcsecondscale relic extension $\sim 20^{\prime \prime}$ east of the nucleus (Baum et al. 1990). Owsianik et al. (1998) have shown that the bright component of $0108+388$ is a very young source with a kinematic age of 367 years. To explain the diffuse structure of $0108+388$ they have adopted a scenario of recurrent activity in the nucleus and have proposed an interpretation of the asymmetries of the extended emission as being caused by light travel time effects.

Contrary to this, Baum et al. (1990) have suggested that $0108+388$ could be a normally aged radio galaxy in which most of the radio emitting plasma is unable to escape from the nuclear region. Such a situation might arise if the host galaxy has recently swallowed a gas rich companion, that has smothered the source. The idea of a recent merger event is also a very plausible explanation of the misalignment between the active (inner) and inactive (outer) parts of the source.

$0108+388$ is the first known example of a CSO with an arcsecond-scale structure. Moreover, it has also been classified as a GPS source because of a spectral turnover, which, according to Marr et al. (2001), results from free-free absorption by nonuniform gas, possibly in the form of a disk in the central tens of parsecs. Instabilities in such a disk could result in a periodic infall of gas, that would produce apparent renewed activity. Carilli et al. (1998) have also found significant H I absorption along the line of sight to the core of $0108+388$, suggesting the existence of a large amount of thermal gas.

Another example of a CSO possessing a (relatively) largescale structure is $0402+379$ (Maness et al. 2004). It has an arcsecond-scale, core-dominated structure and has been 

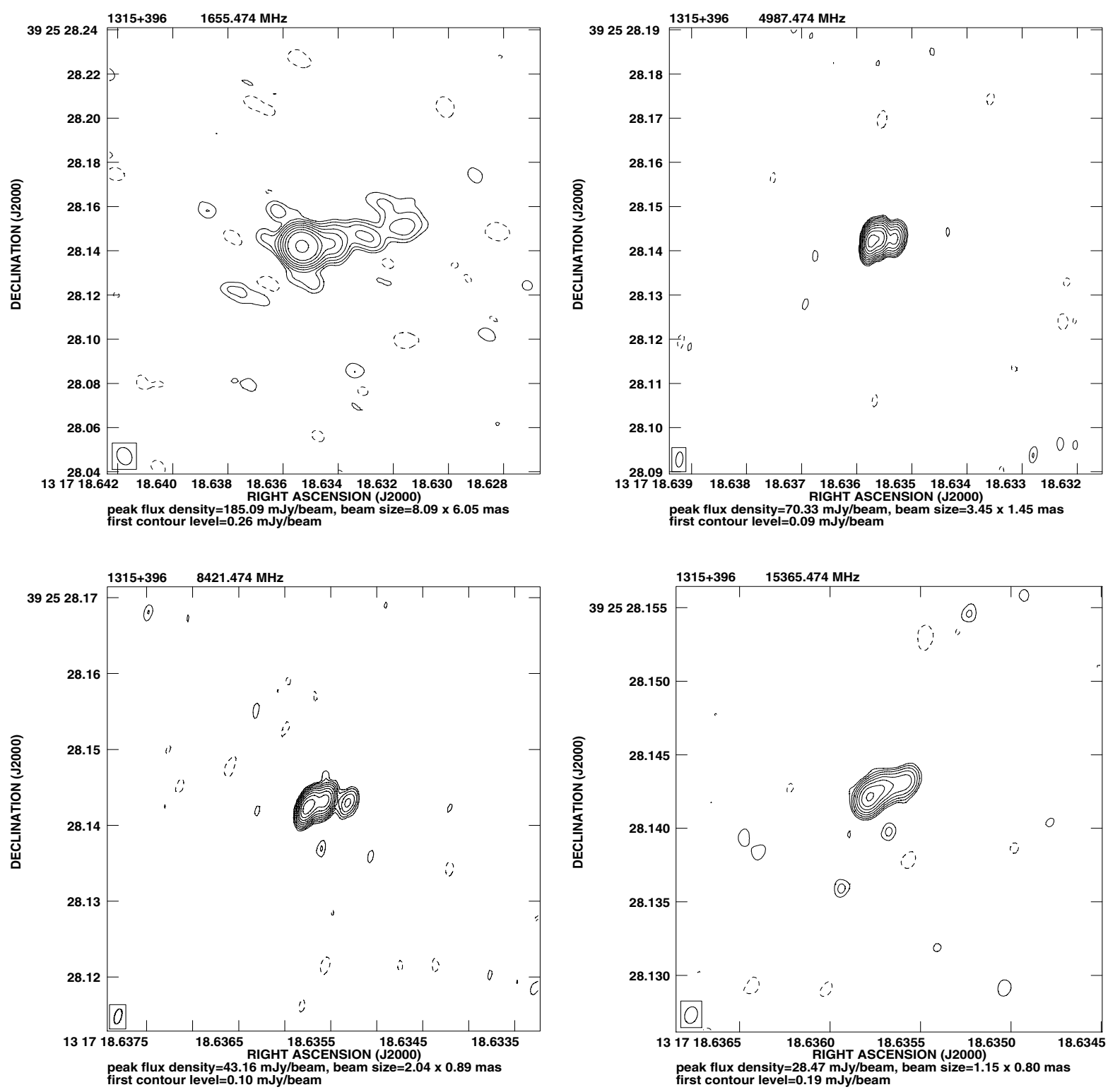

Fig. 5. The VLBA maps of $1315+396$ at 1.65, 5, 8.4 and $15.4 \mathrm{GHz}$. Contours increase by a factor 2 and the first contour level corresponds to $\approx 3 \sigma$.

classified as an MSO. Unlike $0108+388$, the outer lobes of $0402+379$ are symmetric.

\subsection{The case of $0809+404$}

As the VLA images of 0809+404 (F2001) show it to have a very asymmetric double structure with 1'.2 separation and a flux density ratio $\sim 100: 1$ at $4.9 \mathrm{GHz}$, we suggest that, as far as the arcsecond structure is concerned, 0809+404 resembles $0108+388$. The weaker western component of the $0809+404$ VLA structure is a relic of previous activity and its spectral index, calculated from the 4.9 and $8.5 \mathrm{GHz}$ VLA observations, is very steep $(\alpha=-1.5)$. This component is not present in the $15-\mathrm{GHz}$ VLA image. There is also no indication of a radio core located elsewhere in this image. However, on a milliarcsecond scale, the differences between the images of $0108+388$ (see e.g. Taylor et al. 1996), and ours of 0809+404 become apparent: $0108+388$ is a triple with mini-FR II-like lobes and a core, whereas no clear FR II-like structure is present in 0809+404, although there is a hint of the existence of two lobes in the 1.65-GHz image. Neither a core nor hotspots are observable at any frequency and the whole milliarcsecond-scale structure fades away towards higher frequencies, indicating that activity in the nucleus has switched off.

It can be seen that there is an appreciable $\left(\sim 40^{\circ}\right)$ misalignment between the axis of the inner structure with respect to the outer, relic structure. Perhaps, as in the case of $0108+388$, a recent merger event in 0809+404 is also a likely scenario.

The eastern bright component shows little Faraday rotation but is strongly depolarized (F2001, Fanti et al. 2004). The polarization asymmetry is very common among smallscale CSS sources and can be caused by differences in the gas 

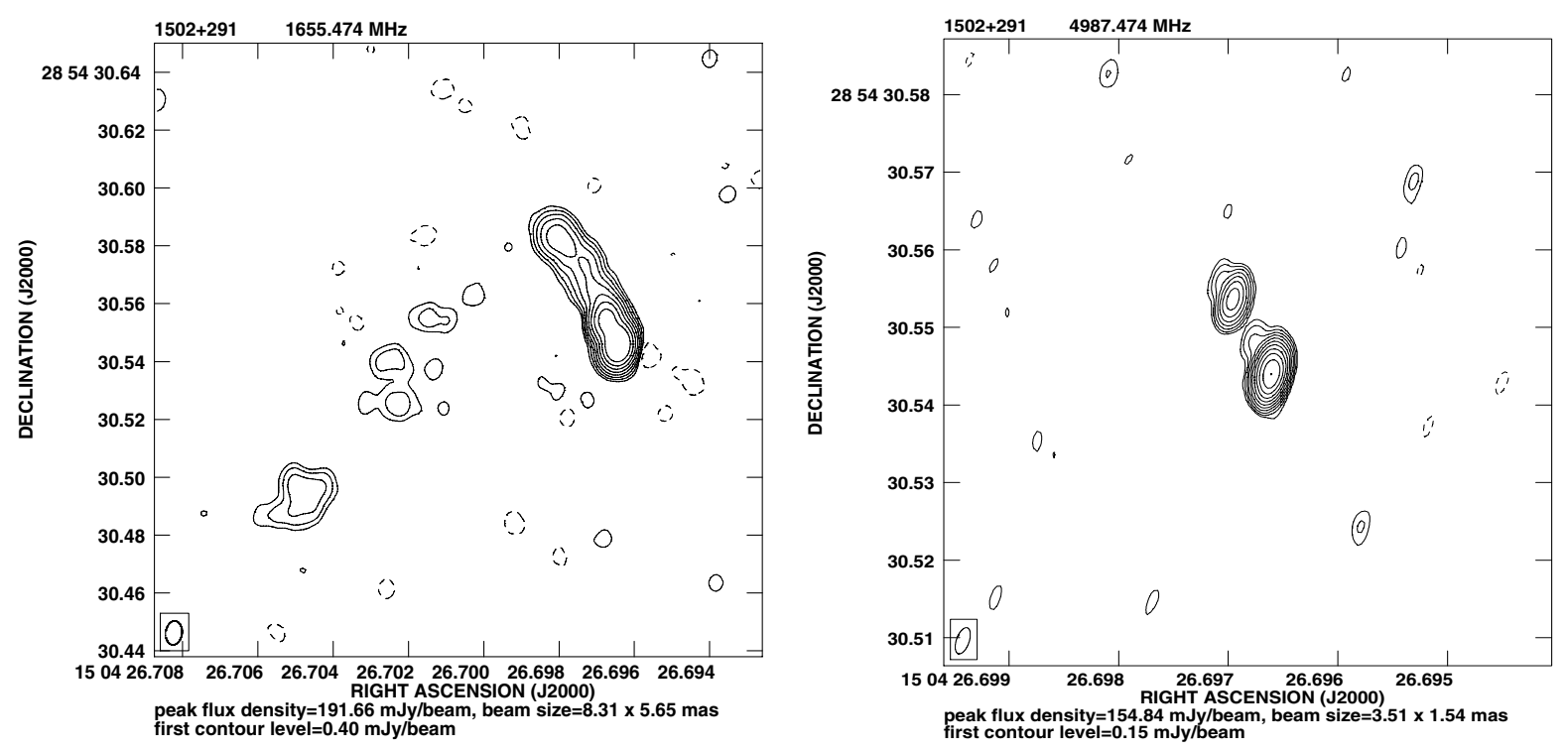

Fig. 6. The VLBA maps of $1502+291$ at 1.65 and $5 \mathrm{GHz}$. Contours increase by a factor 2 and the first contour level corresponds to $\approx 3 \sigma$.

Table 2. Flux densities of sources principal components at observed frequencies.

\begin{tabular}{|c|c|c|c|c|c|c|c|c|c|}
\hline $\begin{array}{c}\text { Source } \\
\text { name } \\
(1)\end{array}$ & $\begin{array}{c}\mathrm{RA} \\
\mathrm{h} \mathrm{m} \mathrm{s} \\
(2)\end{array}$ & $\begin{array}{l}\text { Dec } \\
\circ, 1, \\
(3)\end{array}$ & $\begin{array}{c}S_{1.65 \mathrm{GHz}} \\
\mathrm{mJy} \\
(4)\end{array}$ & $\begin{array}{c}S_{5 \mathrm{GHz}} \\
\mathrm{mJy} \\
(5)\end{array}$ & $\begin{array}{c}S_{8.4 \mathrm{GHz}} \\
\mathrm{mJy} \\
(6)\end{array}$ & $\begin{array}{c}S_{15.4 \mathrm{GHz}} \\
\mathrm{mJy} \\
(7)\end{array}$ & $\begin{array}{c}\theta_{1} \\
\prime \prime \\
(8)\end{array}$ & $\begin{array}{c}\theta_{2} \\
\prime \prime \\
(9)\end{array}$ & $\begin{array}{c}\mathrm{PA} \\
\circ \\
(10)\end{array}$ \\
\hline \multirow[t]{2}{*}{$0809+404$} & 081253.123 & 401859.880 & 287.6 & $193.9 *$ & $74.5 *$ & - & 0.018 & 0.012 & 137 \\
\hline & 081253.124 & 401859.872 & 288.9 & & & - & 0.022 & 0.012 & 149 \\
\hline \multirow[t]{3}{*}{$0949+287$} & 095206.102 & 282832.400 & 199.8 & 29.6 & 8.5 & - & 0.015 & 0.007 & 83 \\
\hline & 095206.079 & 282832.417 & 238.2 & 13.1 & 10.8 & - & 0.020 & 0.014 & 42 \\
\hline & - & - & $238.5 \dagger$ & - & - & - & - & - & - \\
\hline \multirow[t]{3}{*}{$1159+395$} & 120149.964 & 391911.041 & 271.2 & 86.5 & 33.4 & - & 0.008 & 0.006 & 172 \\
\hline & 120149.965 & 391911.003 & 162.6 & 37.7 & 13.6 & - & 0.007 & 0.006 & 52 \\
\hline & 120149.965 & 391911.028 & - & 5.1 & - & - & - & - & - \\
\hline \multirow[t]{2}{*}{$1315+396$} & 131718.632 & 392528.151 & 1.5 & - & - & - & 0.013 & 0.006 & 105 \\
\hline & 131718.635 & 392528.142 & 244.8 & 147.5 & 100.4 & 51.9 & 0.005 & 0.001 & 106 \\
\hline \multirow[t]{4}{*}{$1502+291$} & 150426.697 & 285430.554 & 113.9 & 18.9 & - & - & 0.005 & 0.003 & 27 \\
\hline & 150426.696 & 285430.545 & 209.1 & 200.7 & - & - & 0.002 & 0.001 & 93 \\
\hline & 150426.698 & 285430.581 & 32.1 & - & - & - & 0.015 & 0.003 & 46 \\
\hline & 150426.705 & 285430.494 & 12.3 & - & - & - & 0.014 & 0.010 & 126 \\
\hline \multirow[t]{2}{*}{$1616+366$} & 161823.581 & 363201.813 & 68.1 & 67.1 & 77.1 & 95.6 & 0.014 & 0.003 & 46 \\
\hline & 161823.580 & 363201.802 & - & 20.7 & - & - & - & - & - \\
\hline
\end{tabular}

Column (1): source name in the IAU format; Col. (2): component right ascension (J2000) as measured at 1.65 GHz; Col. (3): component declination (J2000) as measured at $1.65 \mathrm{GHz}$; Col. (4): VLBA+Effelsberg flux density in mJy at $1.65 \mathrm{GHz}$ from the present paper; Col. (5): VLBA flux density in mJy at $5 \mathrm{GHz}$ from the present paper; Col. (6): VLBA flux density in mJy at $8.4 \mathrm{GHz}$ from the present paper; Col. (7): VLBA flux density in mJy at $15.4 \mathrm{GHz}$ from the present paper; Col. (8): deconvolved component major axis angular size at $1.65 \mathrm{GHz}$ obtained using JMFIT; Col. (9): deconvolved component minor axis angular size at $1.65 \mathrm{GHz}$ obtained using JMFIT; Col. (10): deconvolved major axis position angle at $1.65 \mathrm{GHz}$ obtained using JMFIT. * The flux density of the whole source at respective frequency calculated by summing the map's clean components. $\dagger$ The flux density of diffuse matter of the eastern component calculated by summing the respective clean components.

density in the surrounding medium (Thomasson et al. 2003). We have begun a programme using the WSRT to investigate the gaseous medium of 23 sources from the parent sample (Paper I) with known redshifts. So far three of them have been observed during WSRT service time at UHF-high frequencies $(700-1200 \mathrm{MHz})$ and $0809+404$ was one of them.
Approximately $3 \mathrm{~h}$ of observing time were spent on each object, but none of them has shown any HI absorption. For $0809+404$, a $2 \sigma$ upper limit of $\tau \sim 0.005$ for the optical depth has been set based upon a noise level of $\sim 3.7 \mathrm{mJy} / \mathrm{beam}$. The results of these observations will be described in detail in a future paper. 

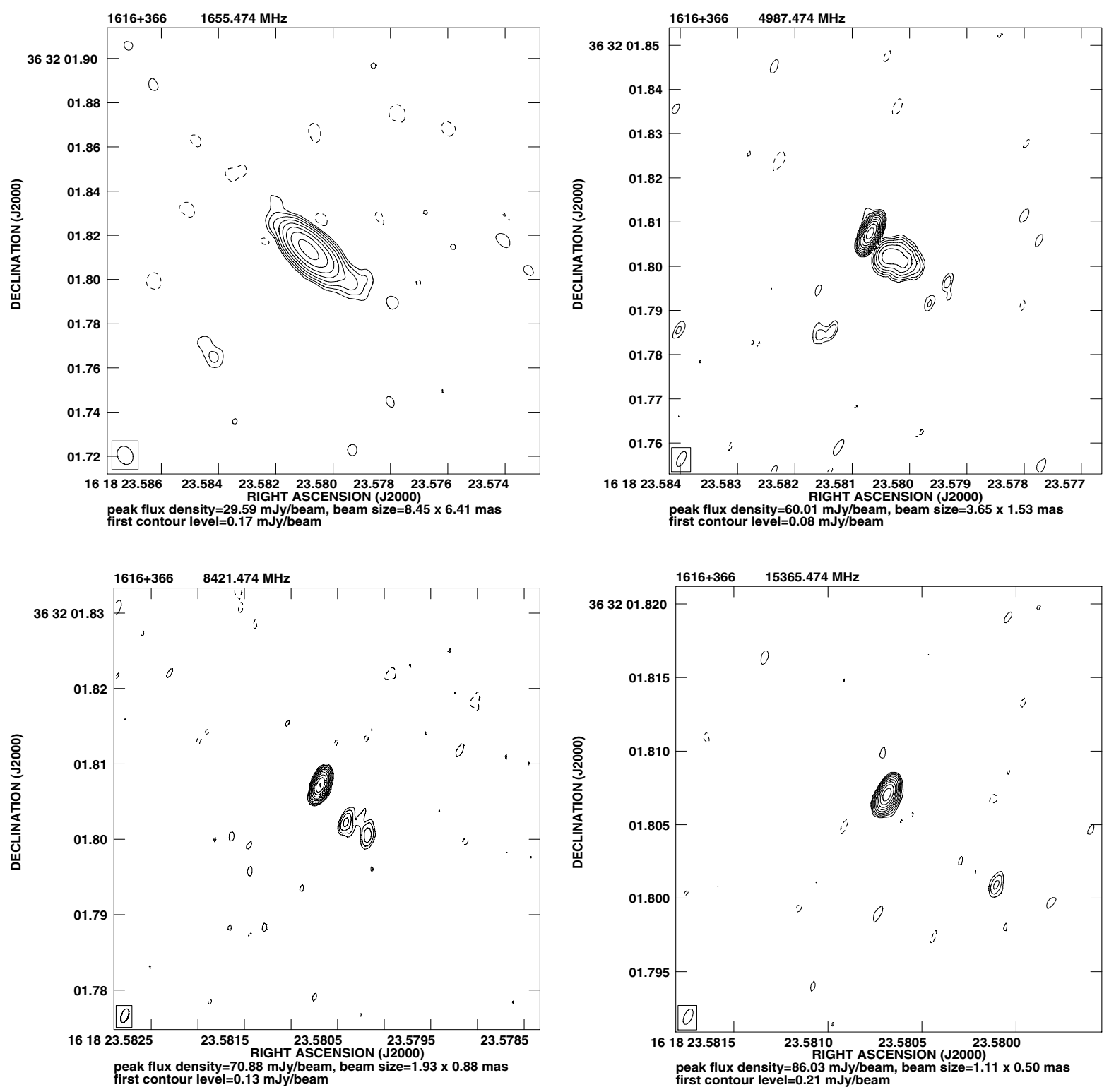

Fig. 7. The VLBA maps of $1616+366$ at $1.65,5,8.4$ and $15.4 \mathrm{GHz}$. Contours increase by a factor 2 and the first contour level corresponds to $\approx 3 \sigma$.

Stanghellini (2003) and Stanghellini et al. (2005) have discussed in detail the properties of a few GPS objects with extended arcsecond-scale emission and have shown that the extended emission around CSO/GPS galaxies is well explained as the co-presence of past and new activity. GPS quasars without a CSO morphology, but having arcsecond-scale emission, are likely to be intrinsically large, old and active radio sources seen along the radio axis, whereas CSO morphologies in quasars, or at least mini-lobe dominated structures, may be signatures of a small and young radio source.

0809+404 is classified spectroscopically as a quasar in SDSS/DR4. More precisely, Zakamska et al. (2003) have found this object to be one of 291 type-II quasar candidates. The spectra of these objects are dominated by narrow emission lines, so their broad-line emission region as well as the
UV-continua are completely obscured at optical wavelengths by a dusty torus. It can be assumed that the axes of such objects are perpendicular to the line of sight. This assumption and the fact that the integrated spectrum of 0809+404 is not of a GPS type (Murgia, priv. comm.) is in agreement with the conjecture that this object is not beamed to the observer. Besides, based on his findings, it is plausible that the inner structure of 0809+404 is younger than the arcsecond-scale emission.

The radio spectrum of $0809+404$ is well described by a continuous injection $(\mathrm{CI})$ model (which refers to the source as a whole) with $\alpha_{\text {inj }}=-0.4$ and a break frequency $\nu_{\text {br }}=1.56 \mathrm{GHz}$ (Murgia, priv. comm.), suggesting that the radio source is continuously replenished by a constant flow of fresh relativistic particles. The initial spectral index $\alpha_{\text {inj }}$, which is the spectral index of the synchrotron radiation in the part of the spectrum 
not affected by the evolution, implies a power law for the energy distribution of the injected electrons of $\delta \approx 1.8$. During the CI phase, the electrons lose energy by synchrotron emission and inverse Compton scattering of the cosmic microwave background photons.

The continuous injection model does not contradict the possibility of intermittent activity, since, during the lifetime of the extended radio emission, the nucleus appears "on average" active. Assuming minimum energy conditions we have estimated a source age $t_{\text {sync }} \sim 10^{5}$ years using the formulae from Miley (1980, Eq. (7)), where $\theta_{x}$ and $\theta_{y}$ correspond to the beam widths of the 4.9-GHz VLA map (produced from the data extracted from the VLA archive). The minimum energy magnetic field was calculated by using Eq. (2) from Miley (1980) and the standard assumptions about the lower and upper cutoff frequencies (10 MHz and $100 \mathrm{GHz}$ respectively), the uniform filling factor $(\eta=1)$, the ratio between protons and electrons $(k=1)$, the angle between the magnetic field and the line of sight $\left(90^{\circ}\right)$ and an equivalent field of the cosmic background radiation $\left(B_{\mathrm{CMB}}=3.25(1+z)^{2}[\mu \mathrm{G}]\right)$. The calculations were made for $4.9 \mathrm{GHz}$ and the corresponding flux density was taken from F2001.

A number of theories trying to explain the episodic activity of radio sources exist in the literature. According to one, activity could be initiated as a result of a merger event. Torques and shocks during the merger can remove angular momentum from the gas in the merging galaxies and this provides injection of substantial amounts of gas/dust into the central nuclear regions (Mihos \& Hernquist 1996). It is, therefore, likely that in the initial phase of an AGN, this gas still surrounds (and possibly obscures) the central regions. Such activity can perhaps cease when there is no more matter to be accreted.

Tingay et al. (2003) have also suggested that at least some GPS sources are limited in their development by the effects of merger activity and the resulting likely sporadic fuelling of the central black holes and accretion disks. Barnes \& Hernquist (1996), who tracked the evolution of both gas and stars in the merger of two disk galaxies, show that in the final state of such a merger, $60 \%$ of the gas is driven into the inner part of the galaxy to within $100 \mathrm{pc}$ of the nucleus. In such an environment, a black hole may undergo many fuelling events and each event may completely disrupt and/or restart the jet. After each renewal, the jet may need to force its way through the dense nuclear environment anew.

Alternatively, to interpret the nature of the inner structure of 0809+404 a theory of the Supermassive Black Hole (SMBH) accretion disk instabilities (Hatziminaoglou et al. 2001; Janiuk et al. 2004, and references therein) could be adopted. It takes into account a non-stationary accretion due to hydrogen ionization that can develop in the disk. These instabilities can cause a sudden accretion of the material surrounding the source and accumulated in the outer torus. It also predicts that galaxies spend the greater part of their lifetime, say $\sim 70 \%$, in a "quiescent" state and $\sim 30 \%$ in an active state with the length of the active phase of an AGN as well as the timescale of the re-occurrence of activity being determined by the mass of the SMBH. Specifically, if the SMBH mass is assumed to be of the order of $10^{7} M_{\odot}-$ and such an assumption is plausible for RLAGNs (Woo \& Urry 2002; Oshlack et al. 2002) - the length of the activity period may be as low as $\sim 10^{3}$ years. This means that the transition to the fader phase can also happen at a very early stage of evolution i.e. at the CSO stage. Note also that such an interpretation is in agreement with an early hypothesis on the episodic nature of CSOs given by Readhead et al. (1994) and recent findings of Gugliucci et al. (2005). However, there is some inconsistency if such an interpretation is applied to $0809+404$, namely that a misalignment between the inner and outer parts of the source is observed. This indicates that the previous period of activity may have been linked to a recent merger. It is to be noted that misalignments between the inner and outer parts of the source can be observed regardless of the source scale. $0809+404$ and $0108+388$ are good examples of very compact sources accompanied by arcsecond-scale relics. In 1245+676, the inner CSO part shows a modest misalignment with respect to the outer megaparsecscale structure (Marecki et al. 2003a) whereas in a few coredominated sources shown by Marecki et al. (2006b) large misalignments of the kiloparsec-scale structures with respect to the outer large-scale ones are quite common.

Another interpretation of the nature of this source is also conceivable, namely that the brighter eastern component of the arcsecond-scale structure is a radio lobe. However, even in this scenario, the lack of a visible hotspot in the eastern, dominating component clearly suggests that the source as a whole is a fader.

\subsection{The other five sources}

The morphology of the edge-brightened lobes of $0949+287$ as seen at $1.65 \mathrm{GHz}$ suggests this source is an FR II like object, with a steep integrated spectrum (Bolton et al. 2004). To calculate its linear size and power we adopted a redshift of $z=1.085$, which is the median value of all the available redshifts of the 60 sources from our primary sample. The estimated linear size and the calculated power at $1.4 \mathrm{GHz}$ (Table 1) indicate that $0949+287$ could be a bright CSS object.

$1159+395$ is a highly redshifted galaxy with a double structure. It is the most powerful object in our sample (Table 1). It appears to be unresolved in the VLA images at 4.9 and $8.5 \mathrm{GHz}$ (F2001) so it can be assumed that it has no extended emission. Its double structure with compact features identified as hotspots as well as a non-GPS spectrum (Murgia et al. 1999) suggest that it is a typical "active" CSO. According to (Murgia et al. 1999), 1159+395 is a very young source with a synchrotron age of the order of $\sim 10^{3}$ years.

The remaining three sources from the subsample presented here $(1315+396,1502+291,1616+366)$ have very compact core-jet structures clearly indicating that these sources are not CSOs so that, even if potentially they could be intermittent, they are in an active phase at the current epoch.

\section{Summary}

Multifrequency VLBA observations of six highly compact yet steep spectrum objects from our parent sample of weak 
CSS sources (Paper I) have been made. The observations presented here (as well as those of Paper II) suggest that some CSS and CSO sources can be short-lived objects. Our results presented so far indicate that fading sources are rare among compact objects with a radio power of order of $10^{26}-10^{28} \mathrm{~W} \mathrm{~Hz}^{-1}$ at $1.4 \mathrm{GHz}$. However, given that small-scale objects are likely to be overpressured (Siemiginowska et al. 2005), the expansion losses would dim a compact source quickly once the central engine switches off. Therefore, such sources are inevitably weaker than their respective "still active" counterparts and, consequently, have largely escaped detection in the GHz-frequency range because of their low radio power. Furthermore, the fader stage of a compact source is rather ephemeral and hence not easily observable. Nevertheless, this selection effect does not preclude the phenomenon of a premature switch-off of a RLAGN from being a direct cause of the small-scale source excess confirmed either statistically (Fanti et al. 1990; O’Dea \& Baum 1997) or observed directly (Gugliucci et al. 2005).

Apart from a natural weakening of the sources, the early cutoff of the energy transport via jets shortly makes the lobes take the form typical for faders, albeit without their spectra showing signs of ageing for frequencies below $5 \mathrm{GHz}$. Therefore, further investigations of LPC sources, i.e. those with radio powers below $\sim 10^{26} \mathrm{~W} \mathrm{~Hz}^{-1}$ at $14 \mathrm{GHz}$ and subarcsecond angular sizes (Giroletti et al. 2005), but not necessarily ultra-steep spectra, might lead to the discovery of more members of compact fader class.

In this context, $0809+404$ appears to be an object of a particular importance. It is a young source with a synchrotron age of the order of $10^{5}$ years, which is typical for CSS objects. However, its morphological structure suggests a more complex past. The weak western component in the VLA image is possibly a relic of previous activity. The bright eastern component appears amorphous with no hotspots in the milliarcsecond scale. Two explanations for the unusual features observed in the VLA (F2001) and our VLBA images are plausible:

1. Restarted activity as an outcome of a merger, shortly followed by a decay of the structures resulting from the new period of activity.

2. Very asymmetric arcsecond-scale double source that is fading away.

Based upon the morphological structures of $0809+404$ seen in the VLA and VLBA images and HI absorption observations, we are leaning to the first interpretation and consider the arcsecond-scale weak western component, analogous to that in $0108+388$, as a signature of a past epoch of activity whereas the dominating eastern component is likely to be a very compact fader. The latter interpretation is consistent with the observational evidence brought by Gugliucci et al. (2005) suggesting that such short periods of activity in AGNs are possible.

Particularly useful would be a high resolution optical image with the sensitivity enabling the search for the evidence of a merger or its aftermath. This could lead to an explanation of the nature of the weak western component. The currently available optical image of $0809+404$ from SDSS/DR4 is not helpful with regard to this.
Acknowledgements. The VLBA is operated by the National Radio Astronomy Observatory (NRAO), a facility of the National Science Foundation (NSF) operated under cooperative agreement by Associated Universities, Inc. (AUI).

Effelsberg telescope is operated by the Max-Planck-Institut für Radioastronomie (MPIfR) and it is a part of the European VLBI Network (EVN).

This research has made use of the NASA/IPAC Extragalactic Database (NED) which is operated by the Jet Propulsion Laboratory, California Institute of Technology, under contract with the National Aeronautics and Space Administration.

Use has been made of the third release of the Sloan Digital Sky Survey (SDSS) Archive. Funding for the creation and distribution of the SDSS Archive has been provided by the Alfred P. Sloan Foundation, the Participating Institutions, the National Aeronautics and Space Administration, the National Science Foundation, the US Department of Energy, the Japanese Monbukagakusho, and the Max Planck Society. The SDSS Web site is http://www.sdss.org/ The SDSS is managed by the Astrophysical Research Consortium (ARC) for the Participating Institutions. The Participating Institutions are The University of Chicago, Fermilab, the Institute for Advanced Study, the Japan Participation Group, The Johns Hopkins University, Los Alamos National Laboratory, the MaxPlanck-Institute for Astronomy (MPIA), the Max-Planck-Institute for Astrophysics (MPA), New Mexico State University, University of Pittsburgh, Princeton University, the United States Naval Observatory, and the University of Washington.

We thank Raffaella Morganti for her help with the WSRT service time observations.

We thank Karl-Heinz Mack for reading of the early version of this paper and a number of suggestions.

This work was supported by Polish Ministry of Education and Science under grant 1 P03D 00830.

\section{References}

Abell, G. O., Corwin, H. G., \& Olowin, R. P. 1989, ApJS, 70, 1

Alexander, P., \& Leahy, J. P. 1987, MNRAS, 225, 1

Alexander, P. 2000, MNRAS, 319, 8

Barnes, J. E., \& Hernquist, L. 1996, ApJ, 471, 115

Baum, S. A., O’Dea, C. P., Murphy, D. W., \& de Bruyn, A. G. 1990, A\&A, 232, 19

Beasley, A. J., Gordon, D., \& Peck, A. B. 2002, ApJS, 141, 13

Bolton, R. C., Cotter, G., Pooley, G. G., et al. 2004, MNRAS, 354, 485

van Breugel, W. J. M., Miley, G. K., \& Heckman, T. A. 1984, AJ, 89, 5

Carilli, C. L, Menten, K. M., Reid, M. J., Rupen, M. P., \& Yun, M. S. 1998, ApJ, 494, 175

Carvalho, J. C. 1985, MNRAS, 215, 463

Cohen, A. S., Röttgering, H. J. A., Jarvis, M. J., Kassim, N. E., \& Lazio, T. J. W. 2004, ApJS, 150, 417

Cordey, R. A. 1987, MNRAS, 227, 695

Dallacasa, D., Tinti, S., Fanti, C., et al. 2002, A\&A, 389, 115 (D2002)

Fanti, R., Fanti, C., Schilizzi, R. T., et al. 1990, A\&A, 231, 333

Fanti, C. 2000, Proc. of the 5th European VLBI Network Symposium held at Chalmers University of Technology, Gothenburg, Sweden, June 29 - July 1, 2000, ed. J. E. Conway, A. G. Polatidis, R. S. Booth, \& Y. M. Pihlström, 73

Fanti, C., Pozzi, F., \& Dallacasa, D., et al. 2001, A\&A, 369, 380 (F2001)

Fanti, C., Branchesi, M., \& Cotton, W. D., et al. 2004, A\&A, 427, 465

Giroletti, M., Giovannini, G., \& Taylor, G. B. 2005, A\&A, 441, 89 
Giroletti, M., Giovannini, G., \& Taylor, G. B., et al. 2003, A\&A, 399, 889

Gugliucci, N. E., Taylor, G. B., Peck, A. B., \& Giroletti, M. 2005, ApJ, 622, 136

Hatziminaoglou, E., Siemiginowska, A., \& Elvis, M. 2001, ApJ, 547, 90

Jamrozy, M., Klein, U., Mack, K.-H., Gregorini, L., \& Parma, P. 2004, A\&A, 427, 79

Janiuk, A., Czerny, B., Siemiginowska, A., \& Szczerba, R. 2004, ApJ, 602,595

Komissarov, S. S., \& Gubanov, A. G. 1994, A\&A, 285, 27

Kunert, M., Marecki, A., Spencer, R. E., Kus, A. J., \& Niezgoda, J. 2002, A\&A, 391, 47 (Paper I)

Kunert-Bajraszewska, M., Marecki, A., Thomasson, P., \& Spencer, R. E. 2005, A\&A, 440, 93 (Paper II)

Liu, R., Pooley, G. G., \& Riley, J. M. 1992, MNRAS, 257, 545

Maness, H. L., Taylor, G. B., Zavala, R. T., Peck, A. B., \& Pollack, L. K. 2004, ApJ, 602, 123

Marecki, A., Barthel, P. D., Polatidis, A., \& Owsianik, I. 2003a, PASA, 20, 16

Marecki, A., Spencer, R. E., \& Kunert, M. 2003b, PASA, 20, 46

Marecki, A., Kunert-Bajraszewska, M., \& Spencer, R. E. 2006a, A\&A, 449, 985 (Paper III)

Marecki, A., Thomasson P., Mack, K.-H., \& Kunert-Bajraszewska, M. 2006b, A\&A, 448, 479

Marr, J. M., Taylor, G. B., \& Crawford, F. III 2001, ApJ, 550, 160

Mihos J. C., \& Hernquist, L. 1996, ApJ, 464, 641

Miley, G. 1980, ARA\&A, 18, 165

Murgia, M., Fanti, C., Fanti, R., et al. 1999, A\&A, 345, 769

O'Dea, C. P., \& Baum, S. A. 1997, AJ, 113, 148

O'Dea, C. P. 1998, PASP, 110, 493

Orienti, M., Dallacasa, D., Fanti C., et al. 2004, A\&A, 426, 463

Oshlack, A. Y. K. N., Webster, R. L., \& Whiting, M. T. 2002, ApJ, 576,81

Owsianik, I., Conway, J. E., \& Polatidis, A. G. 1998, A\&A, 336, L37

Patnaik, A. R., Browne, I. W. A., Wilkinson, P. N., \& Wrobel, J. M. 1992, MNRAS, 254, 655
Phillips, R. B., \& Mutel, R. L. 1982, A\&A, 106, 21

Polatidis, A. G., \& Conway, J. E. 2003, PASA, 20, 69

Readhead, A. C. S., Xu, W., Pearson, T. J., Wilkinson, P. N., \& Polatidis, A. G. 1994, in Compact Extragalactic Radio Sources, NRAO Workshop, ed. J. A. Zenzus, \& K. Kellermann

Readhead, A. C. S., Taylor, G. B., Xu, W., et al. 1996, ApJ, 460, 612

Reynolds, C. S., \& Begelman, M. C. 1997, ApJ, 487, L135

Siemiginowska, A., Cheung, C. C., LaMassa, S., et al. 2005, ApJ, 632, 110

Slee, O. B., Roy, A. L., Murgia, M., Andernach, H., \& Ehle, M. 2001, AJ, 122, 1172

Snellen, I. A. G., Schilizzi, R. T., Miley, G. K., et al. 1999, NewAR, 43,675

Snellen, I. A. G., Schilizzi, R. T., Miley, G. K., et al. 2000, MNRAS, 319,445

Stanghellini, C. 2003, PASA, 20, 118

Stanghellini, C., O'Dea, C. P., Dallacasa, D., et al. 2005, A\&A, 443, 891

Taylor, G. B., Readhead, A. C. S., \& Pearson, T. J. 1996, ApJ, 463, 95

Thomasson, P., Saikia, D. J., \& Muxlow, T. W. B. 2003, MNRAS, 341, 91

Tingay, S. J., Edwards, P. G., \& Tzioumis, A. K. 2003, MNRAS, 346, 327

Vigotti, M., Grueff, G., Perley, R., Clark, B. G., \& Bridle, A. H. 1989, AJ, 98, 419

Vigotti, M., Vettolani, G., Merighi, R., Lahulla, J. F., \& Lopez-Arroyo, M. 1990, A\&AS, 83, 205

Vigotti, M., Vettolani, G., Merighi, R., Lahulla, J. F., \& Pedani, M. 1997, A\&A, 123, 219

White, R. L., Becker, R. H., Helfand, D. J., \& Gregg, M. D. 1997, ApJ, 475,479

Wilkinson, P. N., Polatidis, A. G., Readhead, A. C. S., Xu, W., \& Pearson, T. J. 1994, ApJ, 432, L87

Woo, J. H., \& Urry, C. M. 2002, ApJ, 581, L5

Zakamska, N. L., Strauss, M. A., Krolik, J. H., et al. 2003, AJ, 126, 2125 